\title{
Staat und Freiheit bei F. W. J. Schelling
}

\author{
André Schmiljun \\ ORCID: https://orcid.org/0000-0002-9415-8495 \\ (Humboldt-Universität Berlin, schmiljun@insystems.de)
}

\begin{abstract}
Nur wer Freiheit gekostet hat, kann das Verlangen empfinden, ihr alles analog zu machen, sie über das ganze Universum zu verbreiten. Wer nicht auf diesem Weg zur Philosophie kommt, folgt und tut bloß andern nach, was sie tun; ohne Gefühl, weswegen sie es tun (Schelling 1809, 147)
\end{abstract}

In seiner 31. Münchner Vorlesung zur "Grundlegung der positiven Philosophie“ aus dem Winter 1832/1833 sieht Schelling die Legitimation für die Existenz des Staates nur in einer Funktion: Seine Aufgabe besteht darin, die Freiheit der Individuen zu garantieren (Sandkühler 2005, 197). Schelling eröffnet dazu einen Gegensatz zwischen Positiven und Negativen. Während die Religion, die Wissenschaft und Kunst zur Seite des Positiven gerechnet werden, gehört der Staat auf die „Seite des Negativen. Er ist lediglich conditio sine qua non eines HÖHEREN Lebens. Das wahre Streben nach rechter Freiheit ist eben, den Staat gegen alles höheren Leben zu beschränken; ihn in seinem diesem höhern Leben nur dienenden Verhältnis zu erhalten, nicht ihn aber zum Zweck zu machen“ (Schelling 1833/34, 210). Für Schelling ist die Bestimmung des Staates verknüpft mit einer Güterabwägung zwischen Individuum und Staat, wobei er soziale Aspekte wie etwa Familie und Arbeit und die mit ihr zusammenhängende, neu entstehende bürgerliche Gesellschaft anders als etwa Hegel ausklammert. „Das Positive des Staates“, wie Schraven schreibt, „kann es allein sein, das höhere Leben des Individuums zu fördern“ (Schraven 1989, 41). Die oben erwähnte Passage richtet sich vor allem als Kritik gegen Hegel. Schelling kommentiert Hegels Staatsverständnis mit der Bemerkung, „daß dessen Philosophie mit einer absoluten Vergötterung des Staates“ ende.

Wenn Geschichte, Kunst und Poesie längst verschwunden sein werden, dauert der 
Staat noch fort. Wenn der spekulative Gedanke ganz wiedergeboren ist, so bleibt ihm gegenüber nichts bestehen als der Staat. Denn er ist der reinste Abdruck des spekulativen Gedankens selbst. Man hat in neuerer Zeit der Hegelschen Philosophie mitunter den Vorwurf gemacht, nicht zu Unrecht. Denn wer Hegels Ansicht des Staates kennt, wird darin leicht alles Ansichten des Illiberalismus finden (Schelling 1833/34, 209)

Die Kritik an Hegel ist vor dem Hintergrund Schellings eigener Überzeugung zu verstehen, wonach der staatliche Machtapparat nicht überorganisiert sein sollte. An einer anderen Stelle, nämlich in der "Geschichte zur neueren Philosophie“ (SW I, 10, 126-164) ${ }^{1}$, wirft Schelling Hegel vor, dass in „der Logik (...) nichts Weltveränderndes [liege]. Weiter führt er aus: „Hegel muß zur Wirklichkeit kommen“ (SW II, 1, 232 und I, 10, 129). Der nun von Schelling entwickelte Lösungsvorschlag zielt darauf ab, den Menschen gegenüber einem einvernehmenden, allgegenwärtigen Staat zu schützen. Bereits im „Ältesten Systemprogramm“ aus dem Jahr 1796 heißt es dazu:

Die Idee der Menschheit voran, will ich zeigen, daß es keine Idee vom Staat gibt, weil der Staat etwas Mechanisches ist, so wenig als es eine Idee von einer Maschine gibt. Nur was Gegenstand der Freiheit ist, heißt Idee. Wir müssen also über den Staat hinaus! - Denn jeder Staat muß freie Menschen als mechanisches Räderwerk behandeln; und das soll er nicht; also soll er aufhören (...). Absolute Freiheit aller Geister, die die intellektuelle Welt ins tragen und weder Gott noch Unsterblichkeit außer sich suchen dürfen (Ältestes Systemprogramm, 50).

Staat und Freiheit sind folglich bei Schelling eng miteinander verwoben, wobei Letzteres der Vorzug zu geben ist. Schelling selbst bezeichnet den Begriff als das „A und O der Philosophie“ (Schulz 1988, 11) und räumt der Freiheit damit eine herausgehobene, exponierte Bedeutung in seinem Werk ein.

Wie jedoch versteht Schelling den Begriff der Freiheit? Hierzu findet sich bei ihm keine eindeutige Definition. Er beleuchtet den Begriff sowohl in Hinblick auf naturphilosophische als auch metaphysische wie praktische Fragestellungen und unterstreicht damit die Breite und Komplexität des Themas. Während Schelling in seiner Dissertation, „de malorum origine“2 , über den Ursprung des Bösen, Freiheit noch stark mit Blick auf die Auslegung der Genesis, der sogenannten Geschichte des Sündenfalls, und unter dem Aspekt der Entstehung des Bösen verknüpft, widmet er ein paar Jahre später eine umfassende philosophische Untersuchung dem Thema, die mit dem Titel „Über das Wesen der menschlichen Freiheit und die damit zusammenhängenden Gegenstände“, kurz „Freiheitsschrift“, 1809 erscheint. Seine ersten Ideen aus der Dissertationsschrift bleiben allerdings richtungsweisend für sein Denken. Freiheit bedeutet für ihn zweierlei: zum einen setzt sie den Menschen in die Verantwortung, sich zu sich selbst und seinem

1 Für den Aufsatz wurde die Ausgabe „Ausgewählte Schriften“ von Manfred Frank aus dem Jahr 1985 benutzt.

2 Der ursprüngliche Titel der Dissertation ist aus Platz gründen an dieser Stelle zusammenkürzt worden. Antiquissimi de prima malorum humanorum origine philosophematis Genes. III. explicandi tentamen criticum es philosophicum. Eine deutsche Übersetzung liefert Reinhold Mokroch: Ein kritischer und philosophischer Auslegungsversuch des ältesten Philosophems von Genesis III über den ersten Ursprung der menschlichen Bosheit. 
Umfeld zu verhalten, anderseits, moralisch gesehen, Entscheidungen zu treffen, die gute bzw. böse Implikationen haben können. Zusammengefasst:

Der reale und lebendige Begriff [der Freiheit] aber ist, daß sie ein Vermögen zum Guten und des Bösen sei. Dieses ist der Punkt der tiefsten Schwierigkeit in der ganzen Lehre von der Freiheit, die von jeher empfunden worden und die nicht bloß dieses oder jenes System, sondern, mehr oder weniger, alle trifft (Schelling $1809,148)$

Schellings Anspruch mit der „Freiheitsschrift“ ist es nicht, Freiheit als philosophisch abstrakte Kategorie zu erklären, sondern in ihrer Bedeutung für die Wirklichkeit des Menschen zu erschließen. Für Schelling ist Freiheit auch wesentlich ein Akt der Selbstbestimmung. Er distanziert sich hierbei von den philosophischen Vorarbeiten von Fichte und Kant, weil er der Meinung ist, dass um „das Konkrete der menschlichen Freiheit zu zeigen, (...) der bloße Idealismus nicht hin [reicht]“ (Schelling 1809, 148). Insbesondere wirft er Fichte vor, sich mit seinem Freiheitsbegriff „in Widersprüche und Unstatthaftigkeiten" (Schelling 1809, 134) zu begeben, Kant hingegen, so Schelling, würde mit seiner begrifflichen Trennung von Ding an sich und Erscheinung in seiner ersten Kritik zwar grundsätzlich richtig liegen, allerdings eine sehr negative Auslegung „durch die Unabhängigkeit von der Zeit“ vornehmen.

Trotz der Kritik an Kant übernimmt Schelling dessen Lesart, wonach Freiheit außerhalb der Zeit zu denken ist (Jacobs 2004, 99). Dazu findet sich in der Freiheitsschrift der Satz: „Das intelligible Wesen jedes Dinges, und vorzüglich des Menschen, ist in diesem zufolge außer allem Kausalzusammenhang wie außer oder über aller Zeit" (Schelling 1809, 177). Dieser Satz ist in einem Zusammenhang mit der Tatsache zu verstehen, dass Schelling und Kant das Sein als Wollen deuten und ferner die Meinung teilen, dass ein Wesen, sofern es über einen freien Willen verfügt, als causa noumenon gilt und den Gesetzmäßigkeiten der Natur, also der Zeit und Kausalität, entbunden ist.

In dieser [der Freiheit], wurde behauptet, finde sich der letzte potenzierende Akt, wodurch sich die ganze Natur in Empfindung, in Intelligenz, endlich in Willen verkläre. - Es gibt in der letzten und höchsten Instanz gar kein anderes Sein als Wollen. Wollen ist Ursein, und auf dieses allein passen alle Prädikate desselben: Grundlosigkeit, Ewigkeit, Unabhängigkeit von der Zeit, Selbstbejahung. Die ganze Philosophie strebt nur dahin, diesen höchsten Ausdruck zu finden (Schelling 1809, 146).

Der freie Wille des Menschen ist mit dem Vermögen ausgestattet, die Zeit und Dinge um ihn herum nach Belieben zu gestalten. In einem kurzen Aufsatz zu Schelling gibt Jacobs hierfür ein passendes Beispiel: Habe ich mich dazu entschieden zu studieren, also aus freien Stücken die Aufnahme eines Studiums gewählt, ist davon auszugehen, dass mich mein Wille durch das ganze Studium trägt, ohne dass es einer täglichen Erneuerung 
meines Entschlusses bedarf. ${ }^{3}$ Hier nun liegt ein wichtiger Punkt für das Verständnis von Freiheit. Schelling will darauf hinaus, dass der Mensch kein fremdbestimmtes Wesen ist ${ }^{4}$, sondern in seiner Natur frei ist, selbst zu entscheiden und sich als Subjekt nach eigenen Vorstellungen zu begründen. Der Mensch ist für sein Glück und seinen Abfall, seine Geschicke und seine Rolle in der Geschichte eigenverantwortlich (Jacobs 1995, 126). Schelling drückt diesen Gedanken mit den folgenden Worten aus: „Die freie Handlung folgt unmittelbar aus dem Intelligiblen des Menschen.“ Und weiter heißt es: „(...) denn frei ist, was nur den Gesetzen seines eignen Wesens gemäß handelt und von nichts andrem weder in noch außer ihm bestimmt ist" (Schelling 1809, 177).

Die voran gegangen Ausführungen zeigen, dass Schelling einen starken Begriff der Freiheit fokussiert, der sich schließlich auch in seiner Bestimmung des Staates niederschlägt. Ähnlich wie beim Begriff der Freiheit lässt sich in Schellings Gesamtwerk keine einheitliche, konstante Theorie zum Staat vorfinden, stattdessen variieren seine politischen Äußerungen von einer ablehnenden, negativen bis hin zu einer akzeptierenden, positiven Staatsauffassung. Charakteristisch für Schelling ist zudem, dass er seine Kritik an der politischen Wirklichkeit mit einer Gegenüberstellung von Idealvorstellungen in Form von wirkmächtigen Metaphern wie der Staat als „Mechanismus“ versus „Zweite Natur“ und „Organismus“ oder als „Kunstwerk" anreichert, die in den beiden nachfolgenden Kapiteln diskutiert werden.

Das Thema Freiheit in der Auseinandersetzung mit seinem Staats- und Rechtsverständnis behandelt Schelling in den 1810 gehaltenen Stuttgarter Privatvorlesungen - Anlass hierfür ist wahrscheinlich die besondere Zusammensetzung der Hörerschaft gewesen, wie Müller-Lüneschloss in ihrer Dissertation umfangreich analysiert (Müller-Lüneschloss 2012, 35ff). Freiheit wird von Schelling als entscheidender Baustein für die Gesellschaft gewertet, den der Staat mit seinen Instrumentarien, mit seinen Verfassungsorganen zu schützen hat. Andererseits kann der Staat die Freiheit für seine Bürger nicht erzwingen, sondern ist darauf angewiesen, dass sich die Bürger ihrer Freiheit ermächtigen (SW I, 3, 582). Freiheit hat für Schelling den höchsten Stellenwert. Er fragt daher, wie die Freiheit des Einzelnen, verstanden als Selbstbestimmung und Selbstbehauptung, in ein Verhältnis mit dem Staat, mit einem gesetzlichen Überbau, gebracht werden kann, ohne dass diese an ihrer Qualität verliert. Wie muss ein Staat beschaffen sein, der seinem Volk Freiraum zur Entfaltung gibt, gleichzeitig allerdings Ordnung, Schutz und Sicherheit garantiert? Etwas anders formuliert, allerdings vom

3 Jacobs führt noch ein anderes sehr gutes Beispiel zur Verdeutlichung der Zeit-Freiheit-Debatte aus: Das Lesen eines Textes beansprucht Zeit. Aber das Lesen selbst realisiert sich nicht in jeder Minute neu, sondern durch die Zeit hindurch und dadurch auch in jeder Minute. Der freie Wille, den wir als Grund des Textes denken, trägt das Schreiben und Lesen durch die Zeit hindurch (Jacobs 2004, 100).

4 Schellings Kritik an dieser Stelle hat durch die Neurowissenschaften wieder einen aktuellen Bezug. So behaupten Autoren des Neurokonstruktivismus beispielsweise, dass das Ich von seinem Gehirn abhängt, dass das Ich letztlich nur ein Produkt von Neuronen begreift. Ein ausführlicher Einwand gegen diese Position findet sich in den Arbeiten von Markus Gabriel (Gabriel 2015, 37). 
Inhalt in die gleiche Richtung gehend, drückt sich Schelling aus:

da jene nach außen gehende Thätigkeit (der Trieb), wie nun auch das Ich sich selbst bestimme, ob das Objektive in sich durch das Subjektive, oder das Subjektive durch das Objektive, auf jeden Fall das einzige Vehikel ist, durch welches von dem Ich aus etwas in die Außenwelt gelangen kann, so kann auch durch die Selbstbestimmung jener Trieb nicht aufgehoben werden. Es fragt sich also, in welches Verhältnis durch Sittengesetz der nach außen gehende Trieb zu der ideellen, bloß auf das reine Selbstbestimmen gerichteten, Thätigkeit gesetzt sey (SW I, 3, 581).

Erstaunlich ist auch die Antwort, die Schelling auf seine Frage gleich selbst gibt. Bevor sich der Staat dem Schutz der Bürger zu wenden und Sicherheit garantieren kann, muss er zunächst selbst in seiner Existenz vor Feinden wie beispielsweise vor den Interessen anderer Staaten geschützt werden. Seine Schlussforderungen können vermutlich auch als Indiz eigener Beobachtungen gedeutet werden. Zum Zeitpunkt der „Stuttgarter Privatvorlesungen“ ist Schelling verschiedentlich bereits Zeuge von Krieg und Frieden gewesen. Mehrmals muss er sehen, wie Staaten gegen einander statt friedlicher Lösungsschritte ihre Interessen allein über militärische Interventionen durchsetzen. Die Gefahr eines Krieges ist Schelling zufolge eine der größten Bedrohungen für die Freiheit des Staates und damit für die Freiheit seiner Bürger. Es ist anzunehmen, dass Schelling die politischen Entwicklungen seiner Zeit als ausgesprochen unbeständig, fragil und wechselhaft wahrgenommen haben muss. So schreibt er in seiner Publikation zum „System des transzendentalen Idealismus“:

Es lässt sich also erwarten, daß vorerst bloß temporäre Verfassungen entstehen, welche alle den Keim ihres Untergangs in sich tragen, und weil sie ursprünglich nicht durch Vernunft, sondern durch den Zwang der Umstände gestiftet sind, früher oder später sich auflösen werden, da es natürlich ist, daß ein Volk unter dem Drang der Umstände manche Rechte erst aufgibt, die es nicht auf ewig veräußern kann, und die es früher oder später zurückfordert, wo denn der Umsturz der Verfassung unvermeidlich, und um so gewisser ist, je vollkommener sie in formeller Rücksicht seyn mag, weil, wenn dies der Fall ist, die machthabende Gewalt jene Rechte gewiß nicht freiwillig zurückgibt, welches schon eine innere Schwäche der Verfassung beweisen würde (SW I, 3, 585).

Um diesem düsteren Szenario zu begegnen, schlägt Schelling die sehr moderne Idee eines übergeordneten supranationalen Verbunds vor, der den Frieden unter den Nationen über Vertragsbeziehungen festsetzt. Schelling konstatiert zur Begründung seines Vorschlags im Vorfeld: „Die höchste Verwicklung entsteht durch die Collision der Staaten untereinander, und das höchste Phänomen der nicht gefundenen und nicht zu findenden Einheit ist der Krieg" (SW I, 7, 462). Schellings Lösungsvorschlag erinnert an Kants Überlegungen zum „Ewigen Frieden“. Darin heisst es, dass es Frieden zwischen den einzelnen Staaten nur unter den einzelnen Staaten unter der Prämisse geben könne, dass diese in einen „Friedensbund“ eintreten. ${ }^{5}$ In abgewandelter Form

5 Kant schreibt: „Trotzdem aber bleibt bestehen, daß die Vernunft vom Throne der höchsten, moralisch gesetzgebenden Gewalt herab den Krieg als Rechtsgang schlechterdings verdammt, und den Friedenszustand zur unmittelbaren Pflicht macht. Ein solcher Zustand kann aber ohne 
findet sich dieser Gedanke auch bei Schelling. Ihm zufolge kann keine Staatsverfassung auch nur gedacht werden, die nicht in eine übergeordnete Organisation integriert ist, „eine Föderation aller Staaten, die sich wechselseitig untereinander ihre Verfassung garantiren“. Das Ziel einer solchen Staatengemeinschaft ist es, langfristig Frieden zu sichern, und die Staaten untereinander durch allgemeine Regeln und Gesetze zu disziplinieren, Partikularinteressen unterzuordnen. Durch einen solchen „Völkeraeropag, zusammengesetzt aus Mitgliedern aller cultivierten Nationen“ besteht die Chance, ,jedes einzelne rebellische Staatsindividuum [durch] die Macht aller übrigen" (SW I, 3, 587) an höhere gemeinsame Gebote zu orientieren. An anderer Stelle habe ich dazu bereits ausgeführt, dass Schelling hiermit wichtigen international-politischen Beschlüssen wie beispielsweise dem Völkerbund von 1920 vorausgreift (Schmiljun 2015, 85). Schelling beantwortet also die Frage nach der Freiheit des Einzelnen nicht losgelöst von der Frage von der Verfassung des Staates. Es kann keine Freiheit zur Entfaltung einen „HÖHEREN Lebens" geben, ohne dass der Staat und wiederum die anderen Staaten untereinander die Freiheit des Einzelnen absichern. Freiheit im politischen Kontext meint also zuallererst die Schaffung von intrastaatlichen Zusammenschlüssen, die von der Bedingung abhängen, dass zunächst Völker freiheitlich über die Verfassung ihrer Staaten abgestimmt haben. Nur auf diesem Weg kann eine bindende Staatenstruktur entstehen, die das Vertrauen aller beteiligten Mitglieder genießt.

\section{Der Staat als Mechanismus}

Wie oben bereits im „Ältesten Systemprogramm“ aufgeführt, zeigt sich bereits beim jungen Schelling eine ausgeprägte Skepsis gegenüber dem Staat. Seine Befürchtung ist, dass dieser die Freiheit des Menschen eingrenzt, unterminiert oder gar auslöscht. Die dafür von ihm durchgängig benutzte Metapher lautet „Mechanismus“. Die Metapher der „Staatsmaschine“ ist in der Staatstheorie des 17. und 18. Jahrhunderts ein geläufiges Modell, allerdings von der Wortbedeutung eher negativ konnotiert. So schreibt Fichte beispielsweise vom „Reiben des mannigfaltigen Räderwerks dieser künstlichen politischen Maschine von Europa“ (Fichte I,I 249). Mit der Maschinen-Metapher verbindet sich ein Programm der sozial-politischen Kritik am modernen merkantilistischen und frühkapitalistisch-industriellen System und folglich am utilitaristischen Modell des Staates als Fabrik (Sandkühler 1968, 77). Die politischen Denker der Romantik bewerten den „état machine“ als Gefahr, als Übel für den Menschen, die nur politische Beliebigkeit und Manipulation hervorbringt. Franz v. Baader beispielsweise warnt vor „der gefährliche[n] Torheit unserer Zeiten, gemäß welcher man sich einbildet, beliebig Gesellschaften (Nationen) konstituieren oder auch destruieren zu können, wie man

einen Vertrag der Völker untereinander weder gestiftet noch gesichert werden. Es muß also ein Bund von besonderer Art sein, den man den Friedensbund nennen kann. Zum Unterschied vom Friedensvertrag, der bloß einen Krieg beendet, würde der Friedensbund die Aufgabe haben, alle Kriege auf immer zu endigen“ (Kant 1795, 30). 
Manufakturanstalten etabliert und wieder abrichtet" (Baader 1925, 153).Für Adam Müller ist „der erste Grundirrtum der gangbaren politischen Systeme (...): der Staat ist nicht bloß eine Manufaktur, Meierei, Assekuranz-Anstalt, merkantilistische Sozietät" (Müller 1809, 37-38 $)^{6}$. In seinem 1800 erschienenen Hauptwerk dem „System des transzendentalen Idealismus“, greift Schelling die Metapher der „Staatsmaschine“ (SW I, 1, 586) im Zuge seiner Ausführungen zur Gewaltentrennung und Verfassung auf. Das Bild des Staates als Maschine ist auch bei ihm negativ ausgerichtet. Der Staat ist eine Notkonstruktion, der man sich zwangsläufig mangels besserer Alternativen bedienen muss. In aller Klarheit bringt Schelling diese Kritik in seiner Schrift „Über das Wesen der deutschen Wissenschaft“ zum Ausdruck. Er schreibt: „Zwar es muß sich in der Ausübung finden, daß der Staat durch alle Mittel nie ein Ganzes wird, ja daß jene blinde Nothwendigkeit nicht einmal erreicht wird, aber immer wird der Grund nur in der Unvollkommenheit des Mechanismus gesucht; neue Räder werden eingefügt, die zur Regulierung wieder anderer bedürfen, u. s. f. ins Unendliche" (SW I, 8, 1).

Daneben verwendet Schelling das Bild von der Staatsmaschine auch im Kontext von Staat und Freiheit, konkret um zu betonen, dass der Staat nicht den Einzelnen in seiner Freiheit, in der Entwicklung seiner Persönlichkeit behindern darf. Dieses Motiv taucht ausführlich in Schellings Denkschrift „Über das Wesen der Deutschen Wissenschaft" aus dem Jahr 1807 auf. Ein Kerngedanke seiner Überlegungen lautet: Die Individualität des Menschen darf nicht preisgegeben werden. Es braucht Strukturen, die ein einvernehmliches Miteinander garantieren können. In einer längeren Passage schreibt Schelling dazu Folgendes:

\begin{abstract}
Aller Mechanismus vernichtet die Individualität, gerade das Lebendige geht nicht in ihn ein und ist ihm nichts. Alles Große und Göttliche aber geschieht immer durch ein Wunder, d. h. es erfolgt nicht nach allgemeinen Gesetzen der Natur, sondern nur durch das Gesetz und die Natur des Individuums. Vertilgung der Individualität ist eben die Richtung eines unmetaphysischen, bloß mechanisch geformten Staates (SW I, 8, 12).
\end{abstract}

Ein Grund für die auffallend pejorative Assoziation mit dem Begriff des Staates scheint in Schellings Denklogik zu liegen. Schelling betrachtet die Gemeinschaft nicht vom Allgemeinen und also vom Staat, aus, sondern beginnt seine politischen Überlegungen mit den Bedürfnissen, Anforderungen und Wünschen des Einzelnen. Für ihn ist die Frage von belang, wie der Einzelne sinnvollerweise in das Gemeinwesen integriert werden soll, ohne dass dessen Persönlichkeit durch das Gemeinwesen Schaden nimmt. Denn die persönliche Individualität jedes Einzelnen ist das „Lebendige“ (Schmiljun 2015, 61). Nur sie gestaltet und formt, lässt Kreativität und neue Entwicklungsmöglichkeiten zu. In einem „mechanisch“ geprägten Staat hingegen „gelangen (...) die am wenigsten durch Individualität ausgezeichnet[en], die gewöhnlichen Talente und am meisten aufgezogenen

6 Christian Möckel hat in einem kurzen, sehr gut zu lesenden Aufsatz im „Jahrbuch für Lebensphilosophie" 2008/09 die wesentlichen Punkte von Müllers Staatsauffassung zusammengestellt, die auffallende Parallelen zu Schelling aufzeigen (Möckel 2008, 69-82). 
mechanischen Seelen zur Herrschaft und Leitung der Angelegenheiten“ (SW I, 8, 12).

Schelling verrät allerding in dieser Abhandlung an keinem Punkt, wen er eigentlich mit seiner Kritik am mechanischen Staat genau im Blick hat. Es bleibt bei allgemeinen Verweisen, dass ein solcher Staat auf „Eigennutz“ (SW I, 8, 12) aus sein würde und auch kein anderes Verhältnis zu seinen Bürgern anstrebe, das nicht den größtmöglichen Gewinn sowie "Geld und Gut" einzubringen verspreche. Der Mensch als Person mit samt seiner Individualität wird lediglich als Mittel zur Erhaltung der Staatsgewalt, als existentielle Absicherung zur Finanzmittel- und Güterbeschaffung angesehen. Was Schelling also kritisiert, ist die vorangetriebene Ökonomisierung durch den Staat, die den Menschen nur noch scheinbar als Sache und nicht mehr in seiner „Persönlichkeit“ als das „Höchste“ (SW I, 8, 11) begreift.

\section{Rettung der „verlorenen Einheit": Der (ideale) Staat}

Trotz aller Kritik am Staat überwiegt bei Schelling die Einsicht, sieht man von den Äußerungen im „Ältesten Systemprogramm“ ab, dass es ohne ein übergeordnetes Regelwerk, ohne einen gesetzlichen Rahmen keinen gesellschaftlichen Frieden geben kann. In den „Stuttgarter Privatvorlesungen“ eröffnet Schelling seinem Publikum, dass Mensch und Natur im Grunde eine Gemeinsamkeit haben. Dazu führt Schelling aus:

Der Mensch ist nicht allein in der Welt", bemerkt Schelling, „es gibt eine Mehrheit von Menschen, es gibt ein Menschengeschlecht, eine Menschheit. Wie die Vielheit der Dinge in der Natur nach einer Einheit strebt und in dieser Einheit sich selbst vollendet und gleichsam glücklich fühlt, ebenso auch die Vielheit in der Menschenwelt (SW I, 7, 460).

Schelling bedient sich eines Vergleichs im Anschluss des Zitats: wie die Natur so ist auch der Mensch daran interessiert, seine Einheit zu finden (Schmiljun 2015, 65). Beiden, der Natur und dem Menschen, ist jedoch gemein, dass sie ihren „wahren Einheitspunkt" verloren haben und auf eine Behelfskonstruktion ausweichen müssen (SW I, 7, 461). Im Falle des Menschen liegt die Antwort auf die Frage nach der wahren Einheit auch nicht in „Gott (...)“ (SW I, 7, 461), da dieser für den Menschen Schelling zufolge unerreichbar und verborgen bliebt. "Gott kann nicht mehr ihre [der Menschheit] Einheit sein“, muss der Mensch weiter suchen nach jener „Natureinheit", die aber „wie das Band aller Dinge zeitlich und vergänglich" (SW I, 7, 461). Die angesprochene Behelfskonstruktion und damit Zwischenlösung, die sich der Mensch als vorübergehende Einheit schafft, ist der Staat. Der Staat ist also nicht wahre Natureinheit, sondern „zweite Natur“ für den Menschen und damit auch immer „eine Folge des auf der Menschheit ruhenden Fluchs“. „Da der Mensch nicht Gott zur Einheit haben kann, so muß er sich einer physischen Einheit unterwerfen“ (SW I, 7, 461). Der Staat übernimmt also eine Funktion: in dem er den Menschen zur „zweiten Natur“ wird, kommt ihm die Aufgabe der Friedens- und Freiheitssicherung zu. Der Staat soll auf der Grundlage der Rechtsverfassung Freiheit und Notwendigkeit 
ausgleichen und harmonisieren (Sandkühler 2005, 196). Als „höhere Natur“ erfährt dieser seine Legitimation dadurch, dass er Gerechtigkeit in der Gemeinschaft und Vielfalt der individuellen Interessen installiert und ständig überwacht:

Es muß eine zweite und höhere Natur gleichsam über der ersten errichtet werden, in welcher ein Naturgesetz, aber ein ganz anderes, als in der sichtbaren Natur herrscht, nämlich ein Naturgesetz zum Behuf der Freiheit. Unerbittlich, und mit der eisernen Notwendigkeit, mit welcher in der sinnlichen Natur auf die Ursache ihre Wirkung folgt, muß in dieser zweiten Natur auf den Eingriff in fremde Freiheit der augenblickliche Widerspruch gegen den eigennützigen Trieb erfolgen. Ein solches Naturgesetz, wie das eben geschilderte, ist das Rechtsgesetz, und die zweite Natur, in welcher dieses Gesetz herrschend ist, die Rechtsverfassung (SW III, 583).

Neben dieser Definition des Staates als „zweite Natur“ entwickelt Schelling im Laufe seiner philosophischen Schaffenszeit weitere, deutlich stärkere Idealvorstellungen vom Staat, um sich von der Metapher des Staatsmechanismus abzusetzen. Die Rede ist von der Idee des Staates als Organismus und dem Staat als Kunstwerk. Das in der Biologie gebräuchliche Organismusmodell gewinnt zu Zeiten Schellings in der Staatsphilosophie stark an Popularität ${ }^{7}$ und wird zu einer der häufigsten Metaphern „der Moderne“, ja "fungiert (...) im sozio-politischen Diskurs, seit der Romantik vor allem als Metapher für eine Staatsutopie gegenbürgerlicher Prägung“ (Frank 1982, 156). In den „Vorlesungen über die Methode des akademischen Studiums“ bestimmt Schelling den Staat nicht mehr als bloßen Mechanismus, sondern als „einen objektiven Organismus der Freiheit" (SW V, 313), in dem eine Harmonie der Notwendigkeit und Freiheit gelingt. ${ }^{8}$ „Die vollkommene Erscheinung (...) ist der vollkommene Staat, dessen Idee erreicht ist, sobald das Besondere und das Allgemeine absolut eins, alles was nothwendig, zugleich frei und alles frei Geschehende zugleich nothwendig ist" (SW V, 313 f.) Die hier nun von Schelling hervorgebrachte Idee eines organischen Staats hat seine historischen Vorläufer einerseits bei Fichte, der die Begriffe Staatrechts und Rechtsphilosophie mit Analogien aus der Biologie erklärt, beides möge man wie ein „organisierte[s] Naturprodukt“ (etwa wie bei einem Baum) analysieren (Schmiljun 2015, 55). Anderseits ist Kant als möglicher Ideengeber zu nennen, der in seiner „Kritik der Urteilskraft“ (Hofmann 1999, 119) für einen anderen Weg der Naturerkenntnis plädiert, der sich nicht nur nach streng kausalen Bestimmungen gemäß mathematisch-empirischen Gesetzmäßigkeiten der Physik orientiert. In der Naturbetrachtung, so Kant, haben wir es ebenfalls mit Phänomenen des Organischen und der Lebenszusammenhänge zu tun, die sich nicht in eine kausale oder mechanische Ordnung bringen lassen (Schmiljun 2015, 56). Das Ideal des organischen

7 Zur Vertiefung der biologischen Diskussion im ausgehenden 18. Jahrhundert, siehe Mischer (1997, 59-98).

8 Bereits in Schellings Naturphilosophie spielt der Begriff Organismus eine wichtige Rolle. Schelling zufolge gibt es in der Natur eine positive und eine negative Kraft. Während die positive Kraft Bewegung hervorbringt, behindert die negative Kraft diesen Prozess. Beide Kräfte finden ihre Einheit in einem umspannenden Prinzip eines Organismus, einer sogenannten Weltseele (Sandkühler 2005, 126). 
Staats beruht auf Schellings Vorstellung, dass die Menschen in einem solchen staatlichen Gefüge Zweck an sich sind, genauso wie der Staat Zweck an sich ist. Der Vorteil dieses Theoriegebildes liegt für Schelling darin, dass der Mensch, in dem er als Zweck an sich verstanden wird, frei und selbstbestimmt agieren kann. Die oben bereits erwähnte Gefahr der Unterminierung der Freiheit durch den Staat soll dem Menschen im organischen Staatsmodell erspart bleiben. Denn dieser wird in die Verantwortung genommen, die allgemeine Glückseligkeit, Befriedigung der sozialen Triebe der menschlichen Natur sowie das Zusammenleben der freien, selbstbestimmten Wesen unter den Bedingungen möglichster Freiheit zu garantieren (Zeltner 1954, 177 und SW I, 5, 316). Der Staat als Organismus darf nicht als Mittel zum Zweck verstanden werden. Der Staat als Organismus ist etwas Unbedingtes, der „Von selbst alle Zwecke erfüllen wird (...) wie die Natur nicht ist, damit ein Gleichgewicht der Materie sei, sondern dieses Gleichgewicht ist, weil die Natur ist" (SW I, 5, 316).

Weniger präsent in Schellings Gesamtwerk ist die Metapher vom Staat als Kunstwerk, die er in seinem "System des transzendentalen Idealismus“ entwickelt. Es scheint auf den ersten Blick nicht zwingend selbstverständlich zu sein, warum Schelling gerade diese Form der Analogie wählt. Erst wenn man sich vergegenwärtig, welche Aufgabe die Kunst bei Schelling zumal in der angesprochenen Abhandlung einnimmt, wird der Zusammenhang plausibler. In der Kunst symbolisiert sich für Schelling die beherrschbare, weil selber hervorgebrachte "Synthesis von Natur und Freiheit" (SW I 3 , 619). Bringt ein Künstler ein Kunstwerk hervor, ist dies immer auch ein besonderer Akt des Schaffens, der auf einem Zusammenschluss des bewussten freien Handelns des Subjekts mit der unbewussten notwendigen Gesetzlichkeit der Natur (Dietzsch 1978, 61). Für Schelling hat die Kunst einen herausgehobenen Stellenwert, sie ist ihm sogar das Höchste, weil sie die Befähigung einer „objektiven „Darstellung (...) der Dinge [vermag], wie sie im Absoluten sind“ (SW I, 5, 387). Schelling hebt sie daher auf eine vergleichbar wichtige Stufe wie die der Wissenschaft. Die Kunst wird bei ihm zu einem Instrument zur Erkennung des Seienden (Schmiljun 2015, 62). Selbst die Philosophie ist hierzu nicht in der Lage, wie Schelling im „System des transzendentalen Idealismus“ festhält. Die Kunst ist sogar über der Philosophie anzuordnen (Zeltner 1954, 189).

Aus diesen allgemeinen Bemerkungen zur Rolle der Kunst bei Schelling wird klarer, warum dieser schließlich auch das Ideal des Staates als Kunstwerk bemüht. Wie im Kunstwerk soll sich im Staat die Gegensätzlichkeit zwischen Notwendigkeit und Freiheit, zwischen dem Einzelnen und dem Allgemeinem in einer Symbiose aufgehen. Der wahre Staat soll ein Kunstwerk sein, allerdings nicht im Sinne eines Handwerkes, sondern als bewusst-unbewusstes, und daher frei Geschaffenes (Hofmann 1999, 105). Auch in diesem bildlichen Vergleich bleibt Schelling jedoch abstrakt und nennt keine genauen Ansätze, wie ein solcher Staat beschaffen respektive zu erreichten sein soll. Die Gründe für die Wahl dieser ungewöhnlichen Metapher haben Sandkühler und Hofmann jeweils unterschiedlich erforscht (Schmiljun 2015, 64). 


\section{Schlussfolgerung}

Der Begriff des Staates wird von Schelling uneinheitlich ausgelegt. Es gibt keine zentrale Definition, keine festen Konturen, die auf ein konsistentes Theoriegebilde schließen lassen würden. Auffallend ist allerdings, dass Schelling trotz scharfer Ablehnung, Kritik und Skepsis am Staat diesen als Rettung der „verlorenen Einheit" und Etablierung einer „zweiten Natur“ für notwendig erachtet und daher an ihm festhält, ja sogar Idealvorstellungen vom Staat als „Organismus“ und „Kunstwerk“ vorgibt. Es sind vermutlich die instabilen politischen Verhältnisse zu Beginn des 19. Jahrhunderts, Schellings Gegenwart, die von Kriegen, gesellschaftlichen Unruhen und sogar Revolutionen gekennzeichnet ist und beim Philosophen den Ruf nach einem starken Staat motivieren. Auf diese bemerkenswerte Spaltung bei Schelling verweist bereits Sandkühler in einem prominenten Aufsatz (Sandkühler 1989, 214). Schellings Ringen um eine Bestimmung des Staates ist schließlich durchweg von der Idee getragen, sein zentrales Motiv der Philosophie, die Freiheit des Menschen, einzufordern. Der Staat, sofern er existiert, muss die Freiheit als Grundvoraussetzung seines Bestehens beinhalten.

\section{Literatur}

Baader F. von. 1925. Schriften zur Gesellschaftsphilosophie, hg. von J. Sauter. Jena: Fischer. Dietzsch S. 1978. Friedrich Wilhelm Joseph Schelling. Leipzig - Jena: Urania.

Fichte J. G. 1964. Gesamtausgabe der Bayerischen Akademie der Wissenschaften. Hg. von R. Lauth, H. Gliwitzky, H. Jacob, E. Fuchs, P. Schneider, G. Zöller. Stuttgart-Bad Cannstatt: Friedrich Frommann - Günther Holzboog.

Frank M. 1982. Der kommende Gott. Vorlesungen über die Neue Mythologie, I. Teil. Frankfurt am Main: Suhrkamp.

Gabriel M. 2015. Ich ist nicht Gehirn. Philosophie des Geistes für das 21. Jahrhundert. Berlin: Ullstein.

Hofmann M. 1999. „Über den Staat hinaus. Eine historisch-systematische Untersuchung zu F. W. J. Schellings Rechts- und Staatsphilosophie“. In M. T. Fögen, C. Schott, M. Senn (Hg.), Zürcher Studien zur Rechtsgeschichte, Bd. 37. Zürich: Schulthess Verlag. Jacobs W. G. 2004. Schelling lesen. Stuttgart: Frommann-Holzboog. Jacobs W. G. 1995. „Die Entscheidung zum Bösen oder Guten“. In O. Höffe und A. Pieper

(Hg.), Über das Wesen der menschlichen Freiheit. Berlin: Akademie Verlag (125148).

Kant I. 1795 [1945]. Zum ewigen Frieden. Hg. von S. Carwin und C. W. Hufeland.

Wiesbaden: Limes Verlag.

Mischer S. 1997. Der verschlungene Zug der Seele. Natur, Organismus und Entwicklung bei Schelling, Steffens und Oken. Würzburg: Königshausen und Neumann. 
Möckel C. 2008. „Lebendige ,Idee' kontra toter ,Begriff'. Adam Müller und seine organische Staatsauffassung“. 4. Jahrbuch für Lebensphilosophie des 18. und 19. Jahrhunderts, hg. von R. J. Kozljanič. München: Albuena Verlag (69-82).

Müller A. H. 1809. Elemente der Staatskunst. Öffentliche Vorlesung von Sr. Durchlaucht dem Prinzen Bernhard von Sachsen-Weimar und einer Versammlung von Staatsmännern und Diplomaten im Winter 1808/1809, Bd. 1. Berlin: Sander.

Müller-Lüneschloss V. 2012. Über das Verhältnis von Natur und Geisterwelt. Ihre Trennung, ihre Versöhnung, Gott und den Menschen. Stuttgart: FrommannHolzboog.

Sandkühler H. J. 2005. Handbuch des Deutschen Idealismus. Stuttgart - Weimar: Verlag J. B. Metzler.

Sandkühler H. J. 1968. Freiheit und Wirklichkeit. Zur Dialektik von Politik und Philosophie bei Schelling. Frankfurt am Main: Suhrkamp.

Sandkühler H. J. 1989. „F. W. J. Schelling - Philosophie als Seinsgeschichte und AntiPolitik“. In H.-M. Pawlowski, S. Smid und R. Specht (Hg.), Die praktische Philosophie Schellings und die gegenwärtige Rechtsphilosophie. Stuttgart: Fromman-Holzboog (199-226).

Schelling F. W. J. 1982 (1809¹). „Philosophische Untersuchungen über das Wesen der menschlichen Freiheit und die damit zusammenhängenden Gegenstände (1809)“. In S. Dietzsch (Hg.), Schriften 1804-1812. Berlin: Union Verlag.

Schelling F. W. J. 1957 (1833/341). „Geschichte der philosophischen Systeme von Cartesius bis auf die gegenwärtige Zeit als Übergang zum System der positiven Philosophie. Wintersemester 1833/34“. In A. Hollerbach (Hg.), Der Rechtsgedanke bei Schelling. Quellenstudien zu seiner Rechts- und Staatsphilosophie. Frankfurt am Main: Klostermann.

Schelling F. W. J. 1985. Ausgewählte Werke. Hg. von M. Frank. Frankfurt am Main: Suhrkamp.

Schelling F. W. J. 1977. „Ältestes Systemprogramm des deutschen Idealismus“. In L. Pareyson (Hg.), Schellingiana Rariora. Torino: Bottega d'Erasmo.

Schmiljun A. 2015. Zwischen Modernität und Konservatismus: Eine Untersuchung zum Begriff der Antipolitik bei F. W. J. Schelling (1775-1854). Berlin: HumboldtUniversität zu Berlin.

Schulz W. 1988. „Freiheit und Geschichte in Schellings Philosophie“. In ders. (Hg.), F. W. J. Schelling: Philosophische Untersuchungen über das Wesen der Freiheit und die damit zusammenhängenden Gegenstände. Frankfurt am Main: Suhrkamp (7-26).

Schraven M. 1989. „Philosophie und Revolution. Schellings Verhältnis zum Politischen im Revolutionsjahr 1848“. In Fichte-Kommission der Bayerischen Akademie der Wissenschaften (Hg.), Spekulation und Erfahrung. Texte und Untersuchungen zum Deutschen Idealismus, Bd. 11. Stuttgart: Frommann-Holzboog.

Zeltner H. 1954. Schelling. Stuttgart: Frommann-Holzboog. 
André Schmiljun (Berlin)

\title{
Staat und Freiheit bei F. W. J. Schelling
}

\begin{abstract}
Der Aufsatz untersucht das Begriffspaar Staat und Freiheit bei F. W. J. Schelling. Die These lautet: Obwohl Schelling den Staat ablehnt, scharf als „Mechanismus“ kritisiert und verurteilt, ist dieser aus seiner Sicht der einzige Garant — als „zweite Natur“ — zur Wiederherstellung der „verlorenen Einheit", Sicherung der Freiheit des Menschen und Ermöglichung eines „Höheren Lebens“. Schelling untersetzt dabei seine Feststellung mit wirkmächtigen Metaphern vom Staat als „Organismus“ und „Kunstwerk“, um seine Idealvorstellungen eines politischen Gemeinwesens zu postulieren.
\end{abstract}

Keywords: Schelling; Staat; Freiheit; Politik

Ethics in Progress (ISSN 2084-9257). Vol. 9 (2018). No. 2, Art. \#9, pp. 118-130.

Creative Commons BY-SA 3.0

Doi:10.14746/eip.2018.2.9 